\title{
Drown Alerting, Preventing And Autonomous Rescue System using Ardiuno, Tacticle switche (weight sensors) and Artificial Intelligence
}

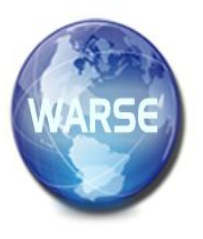

\author{
Pillalamarri. Laxman \\ Lovely Professional University \\ Email: laxmanabcd@gmail.com \\ Prof.Anuj Jain ${ }^{2}$ \\ Lovely Professional University \\ India, \\ Email: a1978jain@gmail.com
}

\begin{abstract}
This paper introduces a novel method of automatic lifesaving swimming pool design to save a drowning victim in a helpless condition by using latest ardiuno(IoT) processor board and the framework. This method utilizes artificial intelligence to save drowning victims without the need of a life-guard(human intervention) from fatal death and also associated specific alerting mechanisms like a loudspeaker. In the present paper, a swimming pool framework is proposed with a responsive elevator assebly surface which is covering entire pool bottom, housing several weight sensitive waterproofed tactical switches arranged to sense any individual or any object
\end{abstract}

incidence(falling) on bottom of the pool ,and associated alerting devices like loud speakers, and a drain motor control. The automatic rescue designs have not been tried succesfull so far in literature effectively so far. The present design delt with simplest algorithm and latest mechanical supporting strucures, and fast responding ardiuno processor. The finished prototype has given promising results in solving defined problem.

Key words: Drowning accident, drowning detection, prevention, screw-jack, ardiuno-board, responsive swimming pool, safety

\subsection{INTRODUCTION}

Drowning is a silent process where a victim cannot shout for help because of water blocking mouth. As soon as the drowning victim drinks enough water and gains weight and reaches the swimming pool floor, the victim's body will settle on the floor due to gravity. After several hours, microorganisms in the stomach and lungs will produce and the dead body will float on water. This floating may take several hours to several days based on water conditions. If the drowned person is taken out of the surface within five or six minutes, survival chances were more without severe brain or organ damage.

A. Roy [1], introduced wearable swimming goggles housing a buzzer and hydrophone backed by an embedded software program. J. Geetha Ramani [2], came up with a solution to this drowning problem by wearable wristband with an inflatable balloon hidden inside a casing, able to work automatically or by the manual trigger. S. Sindhuja [5], has an idea of integrating pressure sensors, GPS to send SMS alert messages, housing an airbag either automatically or by a manual trigger mechanism. [7] Muhammad Ramdhan MS1, Muhammad Ali2 come up with a headband type model with IoT technology integrated by several sensors like a pulse-oxytomertic sensor, RF Signal Detector, GPS for SMS alert. Y. Nishida [9] came up with an idea of leaving a spherical transparent device housing a gyroscope, motion sensor to identify the event of a baby falling in a bathtub or any other small water body indoors. M. A. Hayat [3], [6] Yaswanth Kumar S K; Praveen O K, CAI Xiaoyang [10], L. Fei, W. Xueli [11], Soren Bonderup [12],[14] Ahmad Ilham, Sherly Prastica Della have proposed several methods by using image processing techniques to predict, detect and avoid drowning .In a servey article by Pillalmarri.L et al, has given a wast summary of research papers and patents covering all most all related art. A. Kulkarni [8] has proposed an embedded device housing several sensors to detect a drowning incident.From [15],[16] and provided manufacturer sites mentioned in the references gave an insite into conseving ideas to solve the defined problem.

\subsection{Proposed Method For Responsive Swimming Pool Design}

After a thorough examination of the prior art, a novel autonomous rescue system for swimming pools has been proposed and implimented. Arduino Uno board, an onchip Ardiuno-Baord is used to sense signals and instruct the other electrical components in the system .Main electrical components include screw jack driver motors and loud speaker. The Figure. 1 clearly illustrates step by step operation of the proposed system. 


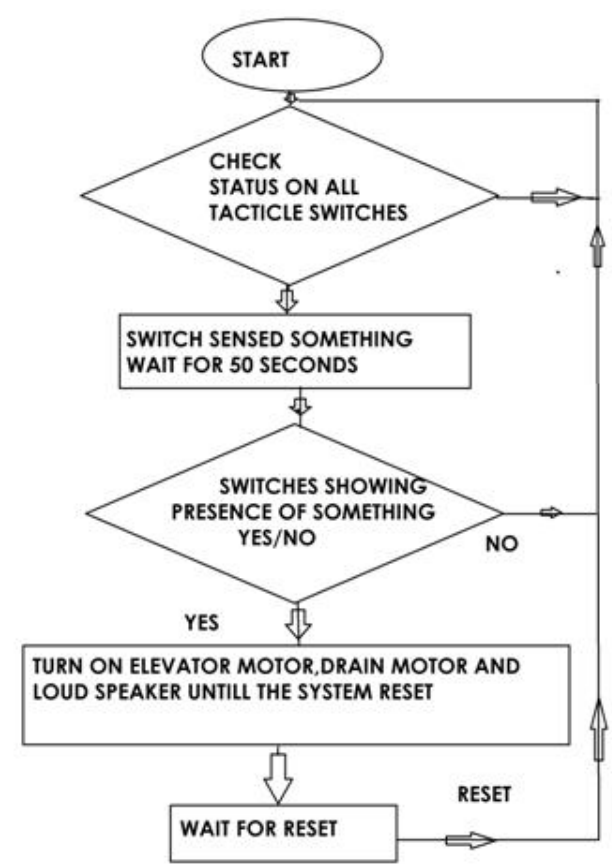

Figure 1.Block Diagram of proposed system

In the prosed design includes a rigid rectangualr swimming pool having regular draining motor used to fill and empty the pool and an added elevator assembly comprising a screw-jack drive assembly and a loudspeaker outside the pool.the said screw-jack assembly includes a powerful high torque electric motor to raise and lower the elevator inside the swimming-pool. Main importantly, the levator surface is covered by an array of pressure sensitive tactical switches to sense incidence of any object on the surface of the said elevator assembly. An array of pressure sensors can also be used in the place of tactical switches with an added library and coding to the existing framework. The total system is controlled by a pre-programmed aurdiuno board .The programming is done according to the given flow-chart in Figure.1. The realisation of the present design not limited to ardiunoboard abut also can be done on a micro-controller based embedded system or any programmable controller framework.

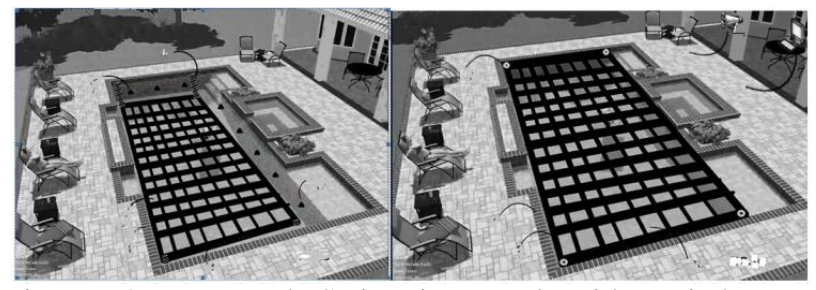

Figure. 2. Proposed Swimming pool with switches arranged all places on bottom elevator -a)lowered b)raised position.

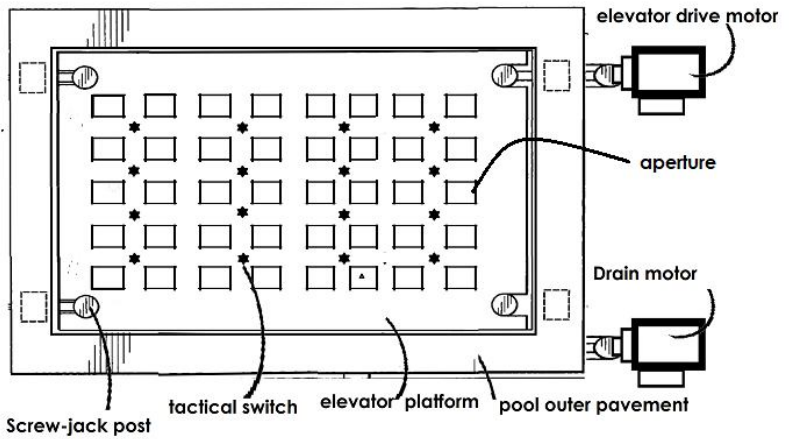

Figure. 3 : Swimming pool (top view) segmentation with a 4x4 switch arrangement and drain motor, apertures

\subsection{Tactical Switches}

The figures shown in Figure.5 illustrates internal function of basic tactical swiches. These switches have 4 basic components such as contact dome base with electrical connections, a plunger covered in a plastic cover .
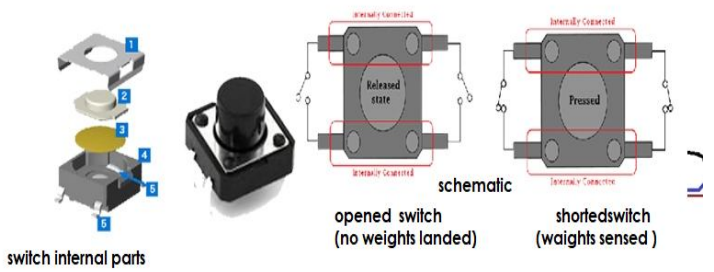

Figure. 4: Illustration of tactile push switch with detailed explanationscematically.

Every switch is monitored continuously by the Ardiunoboard every 10 seconds. The type of trigger switches is shown in Figure. 4 and Figure.5. Once a switch press is detected, the Ardiuno-Baord will start counting the number of switches pressed. If the number of switches pressed continuously by an external force is more than the preset threshold number in that segment, then the Ardiuno-Baord will wait for 50 seconds(decided by programing Ardiuno-Board) based on program. Even after 50 seconds, still, the switches pressed are not changing in that segment, the Ardiuno-Baord assumes a person is unconscious on the swimming pool floor, then a non-mask-able "PANIC"command is generated to initiate rescuw operation .

The RESCUE operation include three actions such as

1) turns $\mathrm{ON}$ the motor corresponding to the respective segment to lift the "Screw Jacks floor(elevator assembly)",

2) powering loud speaker and

3)water-suction drain motor,

By the 3 actions considered as as lifting the drowning victim and alerting near by individuals. As soon as the drown victim assumed as out of the water, the Ardiunoboard will wait for the RESET command manually. Whenever a RESET command given by pressing the RESET button on Ardiuno-board, The " Screw Jacks floor(elevator assembly)" mechanism will go back to the bottom of the swimming pool and water will enter the 
swimming pool normally drain motor subsequently. After this RESET mechanism again Ardiuno-Baord starts checking for any switch press and cycle repeats as shown in flowchart in Figure.1.

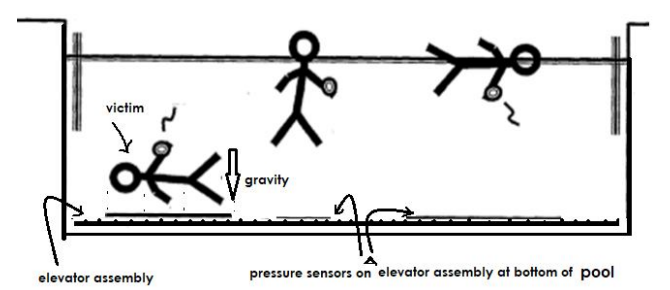

Figure. 5: Showing person at the bottom (victim) pressing switches on the pool floor, and a safe person close to the surface.

In this method, the swimming pool is divided into a single segment or several segments and several electrical toggle switches were placed and connected parallel to the Ardiuno-Baord. Each segment is covered by a " elevator assembly" attached to individual motors which can easily lift to the top of the pool as shown in Figure. 2 and Figure.10. The management of the swimming pool should place a signboard about this system and warn the swimmers not to step on triggers more than 30 seconds accidentally to avoid false alarms.

Figure.6 is a schematic illustration of the proposed system with all parts included, such as elevator assembly in lowered position, a computer (ardiuno-board), drain motor and a loud speaker. The elevator assembly is supposed to have several apertures to allow transmission of water to either direction effortlessly. The said apertures not shown in prototype picture but important to keep in realization.

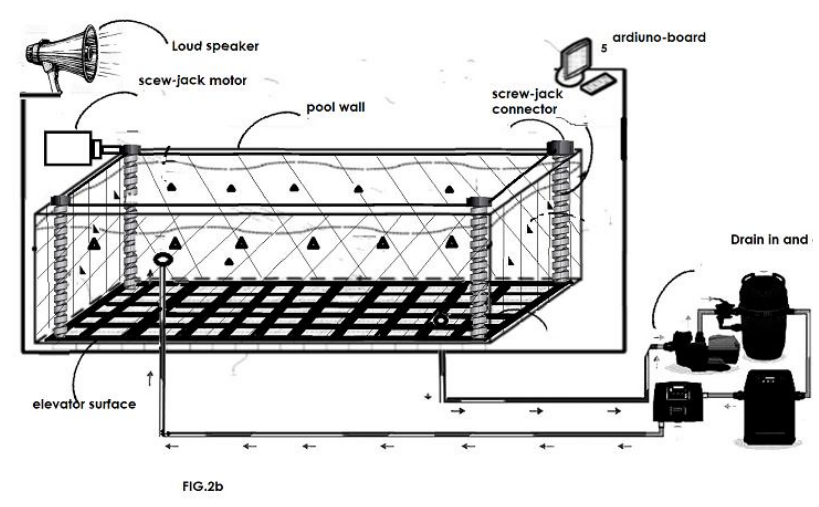

Figure. 6: Graphical representation of Lift mechanism with motor arrangement with "Mechanical Screw Jacks (elevator assembly)" in bottom position and loud speaker arrangement.

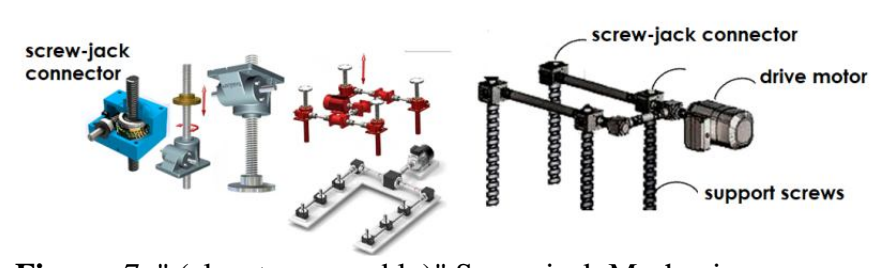

Figure. 7. " (elevator assembly)" Screw jack Mechanism working(two arrangements)with a single motor.

The system in Figure.7 is a special mechanism to easily quickly lift anything in the machine with a single motor efficiently with the help of a Screw jack. The elevator supposed to/programmed to rest on the top surface automatically which is marked OFF at night times or when not in use. This will prevent kids or pets from falling in the pool.

\subsection{Screw Jack mechanism study}

A screw jack is illustrated in Figure.7, in various dimentions and configurations. The screw-jack is wonderfull noise free, durable smooth moving mechanical lifting moving heavy duty apparatus [20].A screw jack is used in applications where linear motion is required, as shown in Figure.8. It is capable of lifting $5 \mathrm{kN}$ to $2000 \mathrm{kN}$ weights in lifting, pulling, and move/align various different loads.Main motto of choosing this mechanical structure is its sychronsation better than any other system[20].

"POWER JACKS" Equipment manufacturer (www.powerjacks.com)[18], has given a scientific formula to calculate speed, torque, and power of uniform elevator assembly supported by screw-jack as follows.

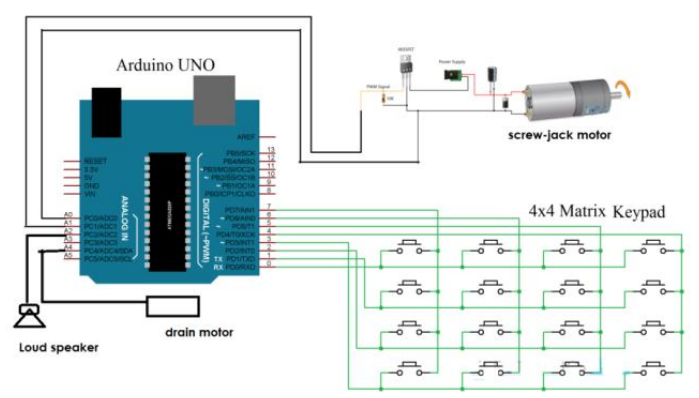

Figure. 8.Prototype of proposed system with a $4 \mathrm{x} 4$ pressure switches and a dc motor to power elevator assembly.

The above logical diagram is phycally realised using iron prototype is displayed in below picture Figure.9(A\&B) . The Figure.9(A\&B) displays both raised and lowered positions. The Figure. 9 ,it has observed that a delay of 20 seconds for both lowering and raising the elevator assembly. 


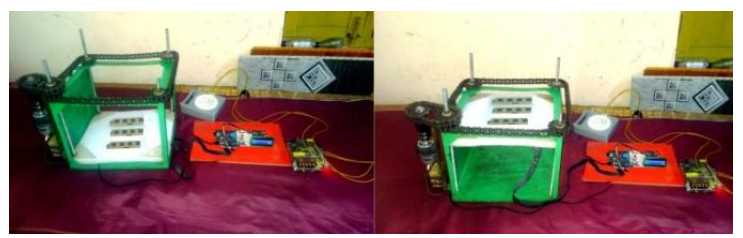

Figure.9A Prototype in LOWERD POSITION Figure.9B Prototype in RAISED POSITION

Figure. 9 is the finished design of proposed design of safe swimming pool. The pool displays a green colored iron frame holding a 4-scews supported screwjack powered by a high torque motor.the elevator assembly is comprising a $4 \times 4$ matrix of pressure sensitive tactical switches to sense any object lying on the elevator surface. An board comprising an LCD panel and a power supply to all the components on the design.

It is clearly observed that, based on the size of the screw and dimention of the pool, upon the sensing of weights on tactical-switch for 50 seconds constantly, the ardiuno-board went into the said PANIC state and ininitiated the resceu operation. The prototype has successfully performed rescue operation in 30 seconds by elevating the (raising) "screw-jack elevator assembly ,turning on drain-motor and powering the said loudspeaker. The elevator assembly used in prototype is made up of light weight synthetic foam to reduce load on the elevator motor.

\subsection{Mathematical Calculations of Screw-Jack Performance}

$$
\begin{aligned}
& \mathrm{N}(\text { Rotations per minute })=[\mathrm{L} * \mathrm{G}] /[\mathrm{P}] \\
& \mathrm{L}=\text { Linear Speed }(\mathrm{mm} / \mathrm{min}) \\
& \mathrm{G}=\text { Gear Ratio } \\
& \mathrm{P}=\text { Pitch }(\mathrm{mm}) \times \mathrm{N}^{\circ} \text { of Starts on Lifting } \\
& \mathrm{Screw} \\
& \text { Relation between screw lead and pitch } \\
& \text { is given as }
\end{aligned}
$$

Note:S= Screw Lead

$$
\mathbf{S}=\mathbf{P} * \text { No of starts }
$$

$\mathrm{P}=$ Pitch

A 8-mm screw was used with a simple nut to realize the proposed framework. Screw-jack is a versitile power amplification and lenear displacement framework.

\subsection{Discussion}

This method is very rugged, hard wired logic and efficient to save accidentally drowning victims from dangerous situations without waiting for a life-guard. Based on the calculations, a suitable motor selected for the given dimentions of swimming pool prototype. The prototype build to represent original idea in a meniaturized form worked well to the given drowning situations . In this design, natural gravitational force is used as a triggering mechanism to togle the tactical switches. A weight sensing sensor can also be used as a sensor with altering program. Simultanesously operating drain-motor has helped the design to quickly reduce the water level so,the victim will take breath quicly. The total design can be implimented with appropriate sensors and heavy duty bulk screw-jack in realtime to build a safe ,responsive swimming pool.

\section{Conclusion}

The proposed method uses rugged and heavy-duty mechanical structures and hard-wired logic and heavy load bearing efficient models. The main duty of the swimming pool is to bring up the victim in an emergency time without compromising anything has been tested by a miniature prototype. The gravitational force, durable tactical switches, packed with powerful Arduino on-chip computer board and mechanical screw jacks together built a novel automatic drown rescue system. Aadapting the proposed system to indoor or outdoor swimming pools will defenitely alliviate fear of swimming among children and oldage people. The realtime swimming pool may have any number of indipendent segments with separate elevators if the swimming pool is very large in size.

\section{REFERENCES}

1 .A. Roy and K. Srinivasan, "A novel drowning detection method for the safety of Swimmers," 2018 20th National Power Systems Conference (NPSC), Tiruchirappalli, India, 2018, pp. 1-6.

2. J. G. Ramani, J. Gayathri, R. Aswanth and M. Gunasekaran, "Automatic prevention of drowning by inflatable wristband system," 2019 5th International Conference on Advanced Computing \& Communication Systems (ICACCS), Coimbatore, India, 2019, pp. 346349.

3. M. A. Hayat, G. Yang, A. Iqbal, A. Saleem, A. Hussain, and M. Mateen, "The Swimmers Motion Detection Using Improved VIBE Algorithm," 2019 International Conference on Robotics and Automation in Industry (ICRAI), Rawalpindi, Pakistan, 2019, pp. 1-6.

4. H. Liu, M. B. H. Frej and B. Wen, "A Novel Method for Recognition, Localization, and Alarming to Prevent Swimmers from Drowning," 2019 IEEE Cloud Summit, Washington, DC, USA, 2019, pp. 65-71.

5. A. Ilham et al., "AuFloat (Autonomous Float) Based-on Artificial Intelligent and LORA (Long Range) Using Haar Cascade Method for Rescuing of Water Accident Victims," 2019, International Symposium on Electronics and Smart Devices (ISESD), Badung-Bali, Indonesia, 2019, pp. 1-4.

6. Y. S. K, P. O. K, and R. R. V, "Autonomous Utility Vehicle (AUVs) Based Emergency Human Drowning Detection System Using Sonar and Thermal Detection Methods," 2019 IEEE International Conference on Distributed Computing, VLSI, Electrical Circuits and Robotics (DISCOVER), Manipal, India, 2019, pp. 1-6.

7. Muhammad Ramdhan MS1, Muhammad Ali2, et al., "An Early Drowning Detection System for the 
Internet of Things (IoT) Applications ", August 2018, TELKOMNIKA, Vol.16, No.4, pp. 1870 1876 ISSN: 1693-6930,

8. A. Kulkarni, K. Lakhani, and S. Lokhande, "A sensorbased low-cost drowning detection system for human life safety," 2016 5th International Conference on Reliability, Infocom Technologies and Optimization (Trends and Future Directions) (ICRITO), Noida, 2016, pp. 301-306.

9. Y. Nishida, K. Hiratsuka, and H. Mizoguchi, "Prototype of Infant Drowning Prevention System at Home with Wireless Accelerometer," SENSORS, 2007 IEEE, Atlanta, GA, 2007, pp. 1209-1212.

10. CAI Xiaoyang, W. Chen and F. Lei, "Application of Image Restoration Based on Robust Estimation in Drowning Warning System," Second Workshop on Digital Media and its Application in Museum \& Heritages (DMAMH 2007), Chongqing, 2007, pp. 33-35.

11. L. Fei, W. Xueli and C. Dongsheng, "Drowning Detection Based on Background Subtraction," 2009 International Conference on Embedded Software and Systems, Zhejiang, 2009, pp. 341-343.

12. Soren Bonderup, Jonas Olsson, "Preventing Drowning Accidents Using Thermal Cameras", 2016, International Symposium on Visual Computing, Advances in Visual Computing pp 111-122. Part of the Lecture Notes in Computer Science book series (LNCS, volume 10073).

13. https://en.wikipedia.org/wiki/Pool_safety_camera
14. S. Sindhuja and P. M. T. Joe, "MEMS based-self regulating airbag drowning aversion system for submerged swimmers," 2015 International Conference on Circuits, Power and Computing Technologies [ICCPCT-2015], Nagercoil, 2015, pp. 1-4.

15. Wenmiao Lu and Yap-Peng Tan, "Swimmer motion analysis with application to drowning detection," 2002 IEEE International Symposium on Circuits and Systems. Proceedings (Cat. No.02CH37353), Phoenix-Scottsdale, AZ, USA, 2002, pp. II-II.

16. H. Eng, K. Toh, W. Yau, and J. Wang, "DEWS: A Live Visual Surveillance System for Early Drowning Detection at Pool," in IEEE Transactions on Circuits and Systems for Video Technology, vol. 18, no. 2, pp. 196210, Feb. 2008.

17.https://www.nsc.org/home-safety/toolsresources/seasonal-safety/drowning 18.[http://www.powerjacks.com/perch/resources/brochur e/pj-sjb-e-bs-en-01b-lowres.pdf]

19. Pillalamarri Laxman, Dr.Anuj Jain, "A State of The Art of Drowning Death Detection and Prevention Systems with Various Communication Technologies and Discussion on their Performance", Conference icsmec2020 29th \& 30th June 2020.

20. www.ludetransmission.com 